\title{
Research on water output optimization of photovoltaic pumping system
}

\author{
Lei Xie $^{1, a}$, Wei Deng ${ }^{1, b}$ \\ ${ }^{1}$ GCL Design \& Research Institute, Suzhou 215124, China

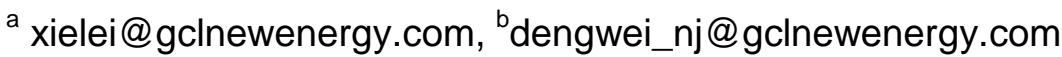

Keywords: PV pumping system, mathematical model, energy optimum utilization, system efficiency, water output

Abstract. This paper focus on the assessment of the influence of insolation conditions \& pump head on the system efficiency and water output of photovoltaic (PV) pumping system, and put forward a kind of PV pumping system with energy optimum utilization, which could optimize the efficiency and water output of PV pumping system. By establishing the mathematical model of the system, the system efficiency and water output of the PV pumping system under different insolation were simulated by using MATLAB/Simulink tools. Some conclusions about the principle of optimum utilization of solar energy, were deduced at the end of this paper.

\section{Introduction}

The applications of PV pumping system provide a feasible way to solve irrigation, drinking water of livestock and inhabitants, especially for some remote areas which power grid cannot extend, such as deserts, islands, mountain areas etc. [1,2]. However, constrained by cost, most of PV pumping systems have not include batteries, which means that when insolation is lower than a certain threshold, the PV pumping system is unable to pump water because of the output power of which could not meet the demand of pump head power, thus reduce the utilization rate of PV pumping system, and lead to solar energy waste. Based on energy optimum utilization thought, this paper summarized some principles which can enhance system performance of PV pumping system, through the way of mathematical model establishing and simulation, evaluation of system efficiency and water output under different insolation condition, pump head and pump switch insolation.

\section{System Configuration}

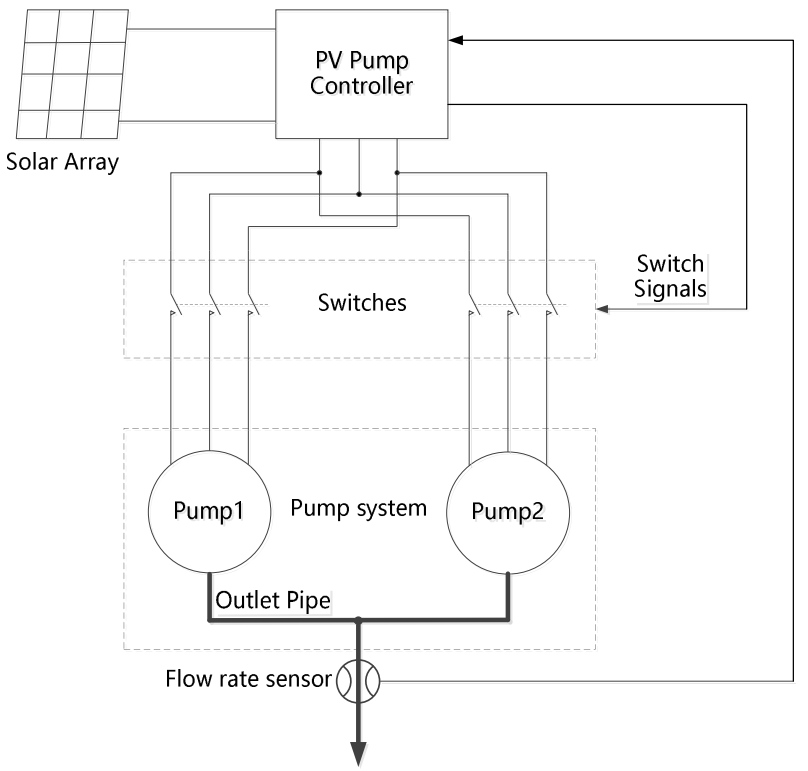

Fig. 1 Structure of PV pumping system with energy optimum utilization

The considered system, shown in Fig. 1, is composed of solar array, PV pump controller, switches, switch signals, pump system, flow rate sensor. For a certain pump, different insolation means 
different flow rate, so pump1 or pump2 can be selected to work according to the flow rate data sampled by flow rate sensor.

\section{Mathematical Model of System}

\section{A. Insolation model}

A sunny day insolation distribution function model was elaborated in this paper for the purpose of simulating system performance [3]:

$$
S(t)=S_{\max } \sin \frac{\pi}{T} t
$$

Where $S_{\max }$ is maximum insolation $\left(\mathrm{W} / \mathrm{m}^{2}\right)$, $\mathrm{T}$ is insolation period, and $\mathrm{t}$ is a certain time of a day.

\section{B. Solar array model}

Solar array is a kind of nonlinear power source, which I-V characteristic can be written as [4]:

$$
I=I_{s c}\left\{1-C_{1}\left[\exp \left(\frac{V+\Delta V}{C_{2} V_{o c}}\right)\right]\right\}+\Delta I
$$

Where $I_{\text {sc }}$ is short circuit current $(A), V_{\text {oc }}$ is open circuit voltage $(V), V$ is working voltage $(V), \triangle$ $\mathrm{I}$ is current increment caused by insolation and temperature $(\mathrm{A}), \triangle \mathrm{V}$ is voltage increment caused by insolation, temperature and series resistance $(\mathrm{V}), \mathrm{C}_{1}$ and $\mathrm{C}_{2}$ are parameters related to solar array characteristic.

\section{Centrifugal pump model}

The head-flow rate (H-Q) characteristic of a centrifugal pump was shown in Fig. 2, The intersection with pipe characteristic under a certain rotation speed is the work point of the centrifugal pump system, and the H-Q characteristic can be described as [5]:

$H(Q)=p_{1} Q^{2}+p_{2} Q+p_{3}$

Where $\mathrm{H}$ is total pump head(m), Q is flowrate $\left(\mathrm{m}^{3} / \mathrm{h}\right), \mathrm{p} 1$, p2, and $\mathrm{p} 3$ are system parameters.

$\mathrm{H}-\mathrm{Q}$ curve of outlet pipe can be written as:

$$
H=H_{s t}+k Q^{2}
$$

Where $\mathrm{H}_{\text {st }}$ is pump head(m), the distance between outlet and water, $\mathrm{k}$ is pipe coefficient, which value is determined by pipe diameter and pipe wall roughness. $\mathrm{k}$ is 0.006 in this paper.

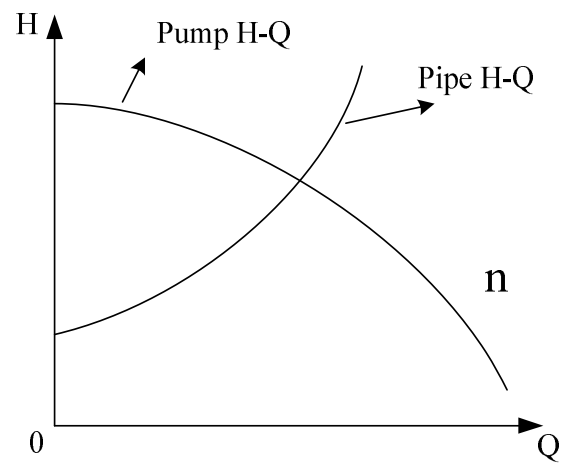

Fig. $2 \mathrm{H}-\mathrm{Q}$ characteristic of pump and pipe 


\section{System Efficiency}

In this paper, the average efficiency was adopted to calculate the whole system efficiency through the way of sampling a great number of instantaneous efficiency data during a period of time, which could be calculated as:

$$
\left\langle\eta_{s}\right\rangle_{\tau}=\frac{\int_{0}^{\tau} P(t) d t}{\int_{0}^{\tau} S(t) d t}=\frac{\int_{0}^{\tau} \eta_{s}(t) S(t) d t}{\int_{0}^{\tau} S(t) d t}=\frac{W_{\tau}}{\sum S_{\tau}}
$$

Where $\left\langle\eta_{s}\right\rangle_{\tau}$ represents the average efficiency of interval $\tau, W_{\tau}$ refers to the total power energy of system $(\mathrm{J}), \sum S_{\tau} \longrightarrow$ is the sum of insolation energy derived from solar energy $(\mathrm{J})$.

\section{Curve Fitting and Simulation}

A. Water pump characteristic curve fitting

Two types of pumps, R95- DF-04 and R95-BF-06 manufactured by RuiRong motor pump industry CO. LTD. Jiangmen, Guangdong province of China, were selected for research in this paper, which characteristics were shown in Table 1[6]. The experimental data were shown in Fig. 3(a) and Fig.3(b).

Table 1 The H-Q characteristcs of two pumps

\begin{tabular}{ccccccc}
\hline Type & \multicolumn{3}{c}{ R95-BF-06 } & \multicolumn{3}{c}{ R95-DF-04 } \\
\hline $\mathrm{H}(\mathrm{m})$ & 20 & 28 & 36 & 12 & 17 & 22 \\
$\mathrm{Q}\left(\mathrm{m}^{3} / \mathrm{h}\right)$ & 7 & 6 & 5 & 10 & 8 & 6 \\
\hline
\end{tabular}

Convenience for analyzing the working conditions of system, according to Eq. (3), this paper used Matlab function-Polyfit to calculate the curve fitting coefficients, the results were as follows:

Table 2 Curve fitting coefficients of R95-DF-04

\begin{tabular}{cccc}
\hline $\mathrm{f}(\mathrm{Hz})$ & $\mathrm{p} 1$ & $\mathrm{p} 2$ & $\mathrm{p} 3$ \\
\hline 50 & -0.1774 & 0.2419 & 26.9093 \\
45 & -0.1534 & -0.0064 & 24.0396 \\
40 & -0.1595 & -0.1350 & 19.9674 \\
35 & -0.0776 & -1.0692 & 18.0374 \\
30 & -0.0902 & -0.8493 & 13.8912 \\
25 & -0.1412 & -0.1314 & 7.9807 \\
\hline
\end{tabular}

Table 3 Curve fitting coefficients of R95-BF-06

\begin{tabular}{cccc}
\hline $\mathrm{f}(\mathrm{Hz})$ & $\mathrm{p} 1$ & $\mathrm{p} 2$ & $\mathrm{p} 3$ \\
\hline 50 & -1.3674 & 8.8560 & 22.5728 \\
45 & -1.4682 & 8.8776 & 18.6202 \\
40 & -1.0948 & 3.0531 & 29.9607 \\
35 & -0.6790 & -0.4241 & 28.8462 \\
30 & -0.6891 & 0.0307 & 19.9336 \\
25 & -0.1756 & -3.3077 & 19.3541 \\
\hline
\end{tabular}


Utilizing Matlab function: Polyval and Plot, the fitting curves were depicted in Fig. 3(a) and Fig. 3(b).

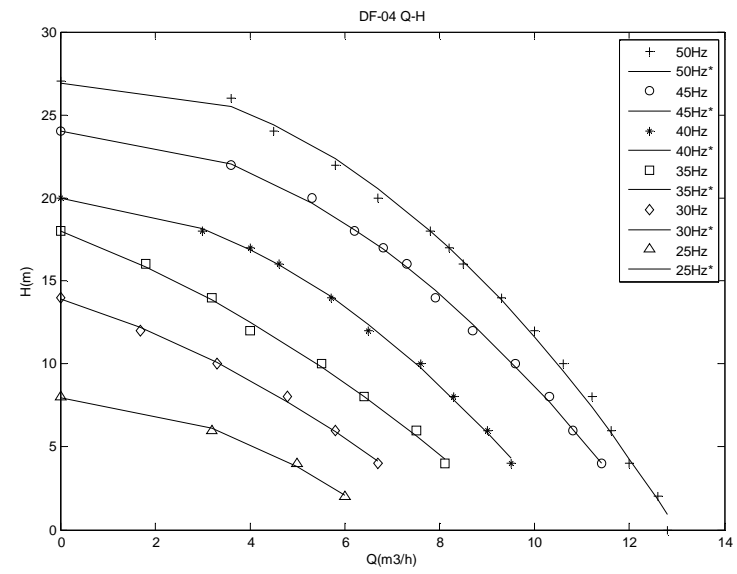

Fig.3（a） H-Q fitting curve of R95-DF-04

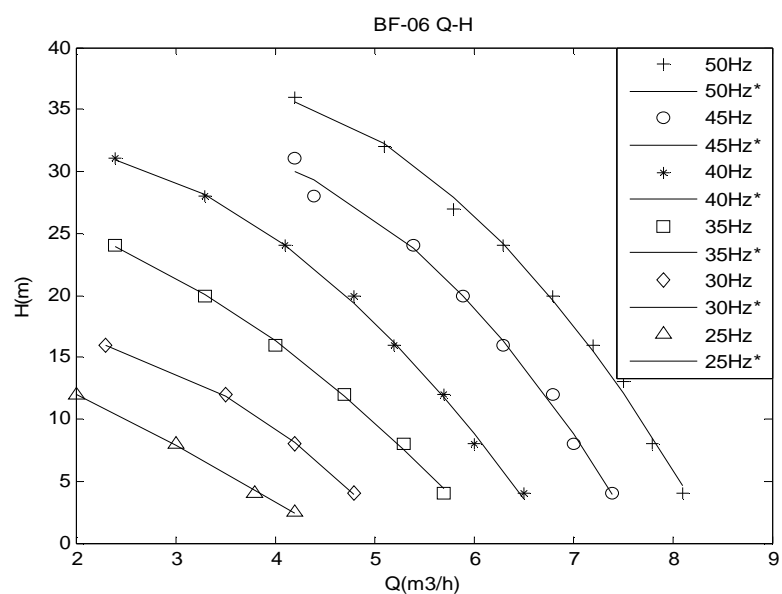

Fig.3（b） H-Q fitting curve of R95-BF-06

\section{B. Simulation}

For analyzing the impact of insolation, pump head to system efficiency and water output, the system model was established in Simulink which was shown in Fig. 4. Three kinds of models, include DF-04 system, BF-06 system, and the proposed system in this paper, were included in this model. System efficiency and water output per day under different insolation conditions, pump heads and pump switch insolation were simulated in this model, the results were as follows:

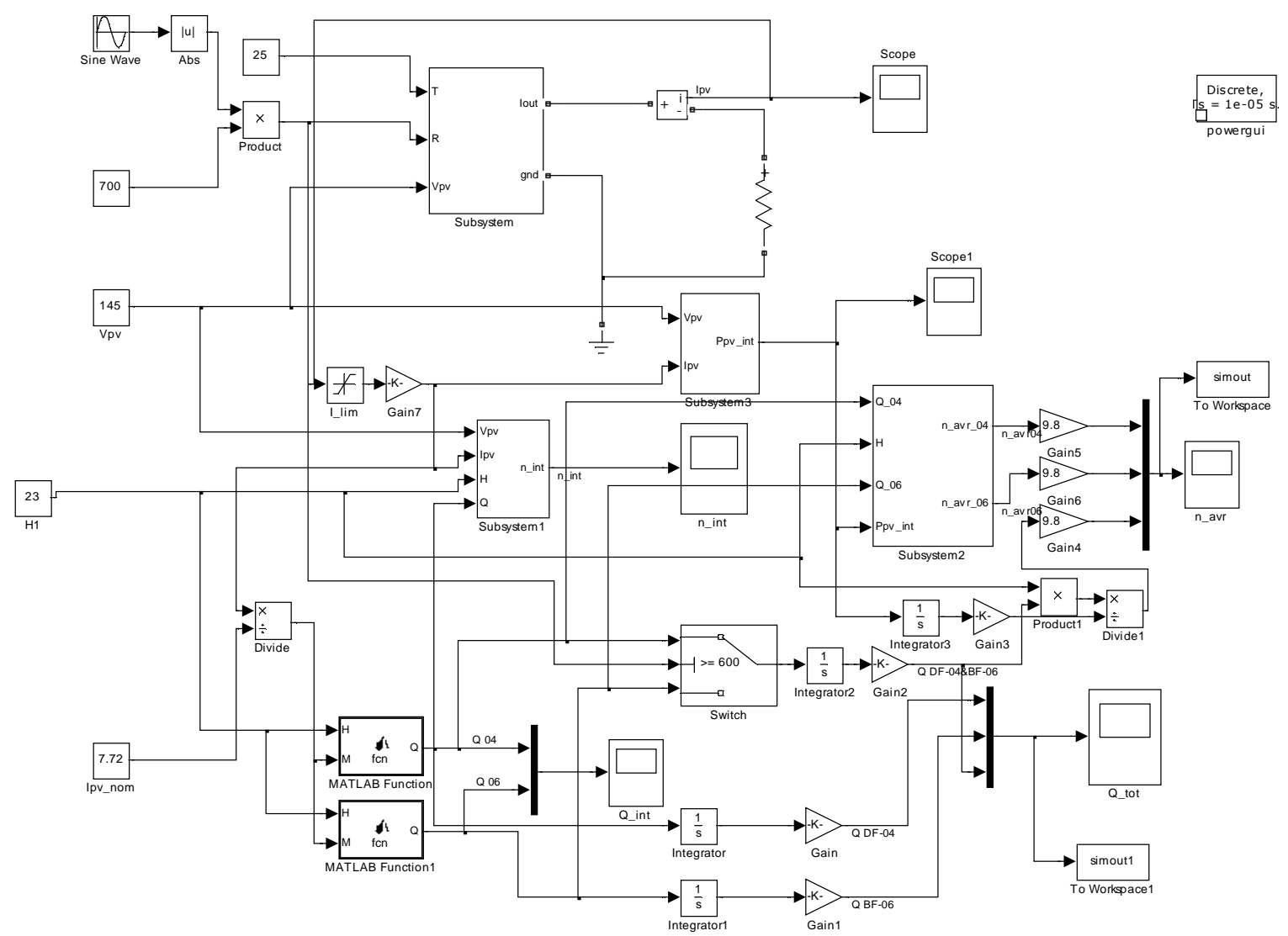

Fig. 4 Simulink model of PV pumping system 
a) System efficiency

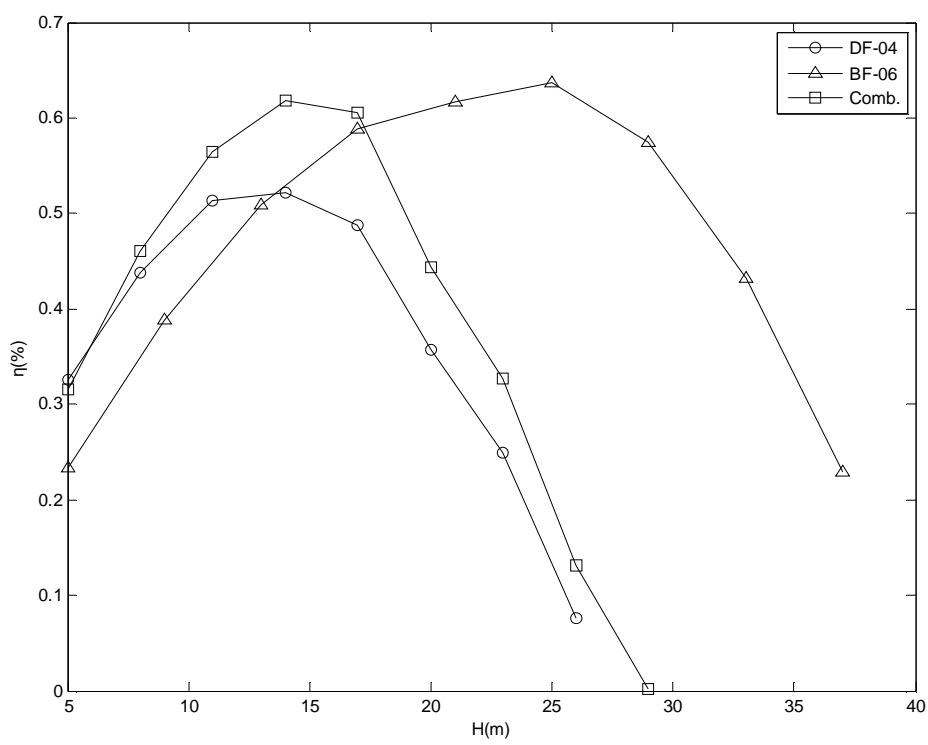

Fig. 5 System efficiency under $S_{\max }=1000 \mathrm{~W} / \mathrm{m}^{2}$

b) Water output per day

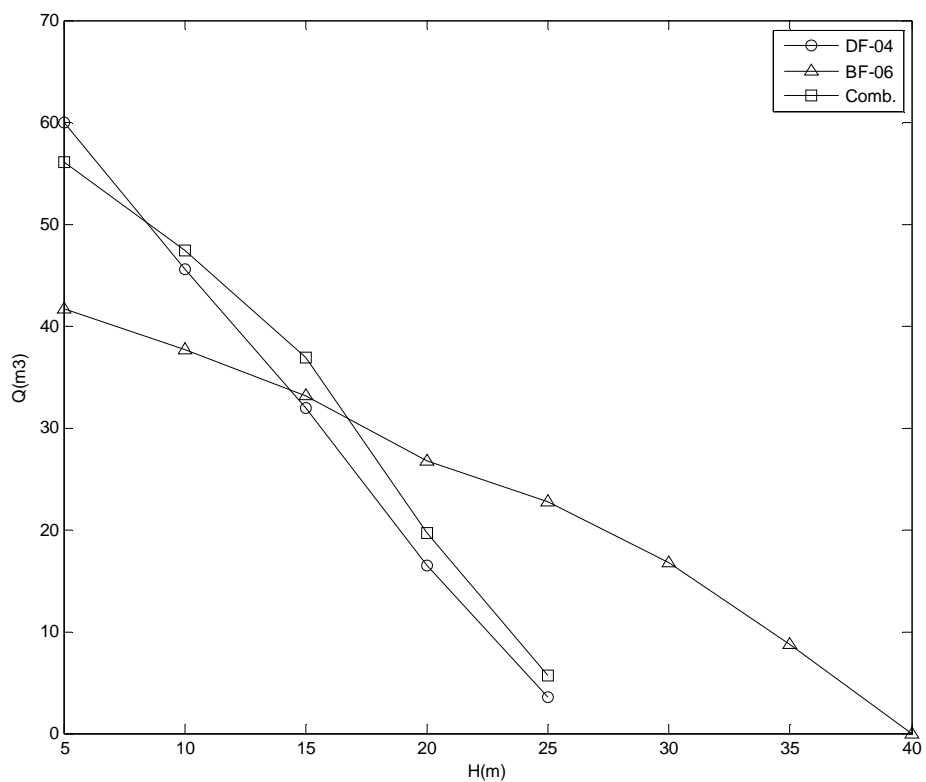

Fig. 6 Water output $\left(\mathrm{m}^{3} /\right.$ day $)$ under $S_{\max }=1000 \mathrm{~W} / \mathrm{m}^{2}$

Fig. 5 and Fig. 6 illustrated the simulation result of system efficiency and water output per day of DF-04 system, BF-06 system and the proposed system(Comb.) in this paper separately under different pump head, with the condition of $S_{\max }=1000 \mathrm{~W} / \mathrm{m}^{2}$, and pump switch insolation is $800 \mathrm{~W} / \mathrm{m}^{2}$.

As can be seen from Fig. 5, when pump head is below than $16 \mathrm{~m}$, the system efficiency of DF-04 system is higher than BF-06 system, the efficiency of the proposed system is higher than BF-06 system when pump head is below than $18 \mathrm{~m}$. And almost in the whole pump head range, the proposed system efficiency is higher than DF-04 system.

As shown in Fig. 6, the regularity of the water output per day of three kinds of water pump systems is similar as system efficiency. So water output per day can be used to evaluate the performance of PV pump system. 


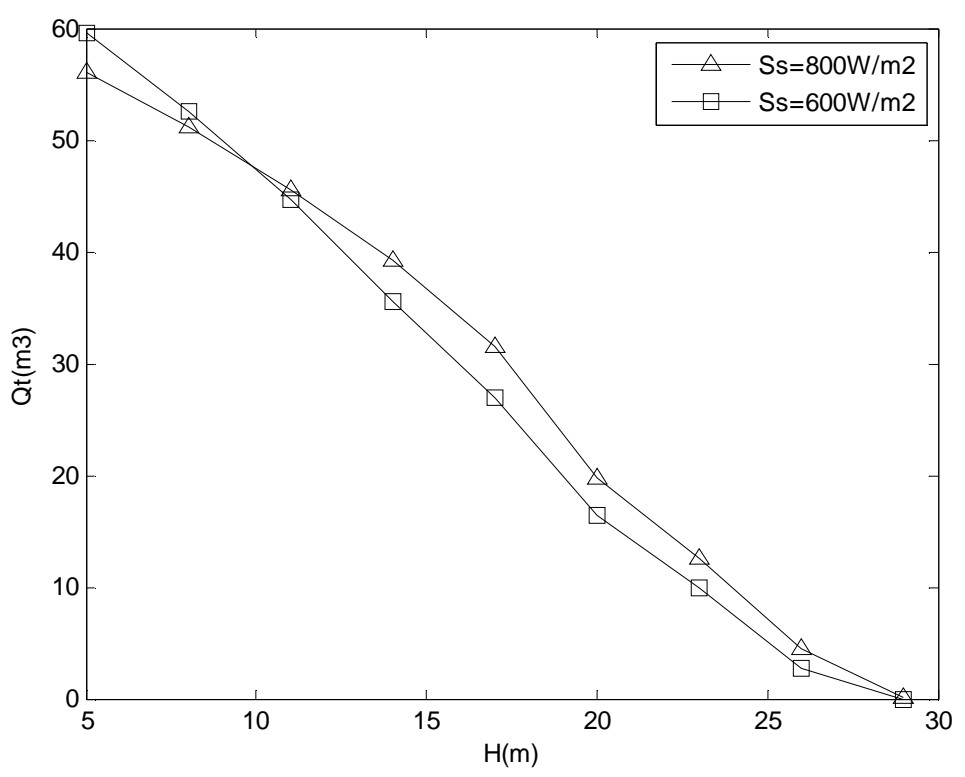

Fig. 7 Water output $\left(\mathrm{m}^{3} /\right.$ day $)$ under different switch insolation

Fig. 7 illustrated the comparison of water output per day of the proposed system under $\mathrm{S}_{\max }=1000 \mathrm{~W} / \mathrm{m}^{2}$ and different pump switch insolation $\left(\mathrm{S}_{\mathrm{s}}\right)$ according to different pump head.

The result showed that when pump head is below than $10 \mathrm{~m}$ with pump switch insolation equal to $600 \mathrm{~W} / \mathrm{m}^{2}$, the water output per day of the proposed system is higher than the situation with pump switch insolation is $800 \mathrm{~W} / \mathrm{m}^{2}$. When pump head is higher than $10 \mathrm{~m}$, the situation is opposite.

Fig. 7 gave out the comparison result of water output per day in the condition of $S_{\max }=700 \mathrm{~W} / \mathrm{m}^{2}$, and pump switch insolation is $600 \mathrm{~W} / \mathrm{m}^{2}$ under different pump head.

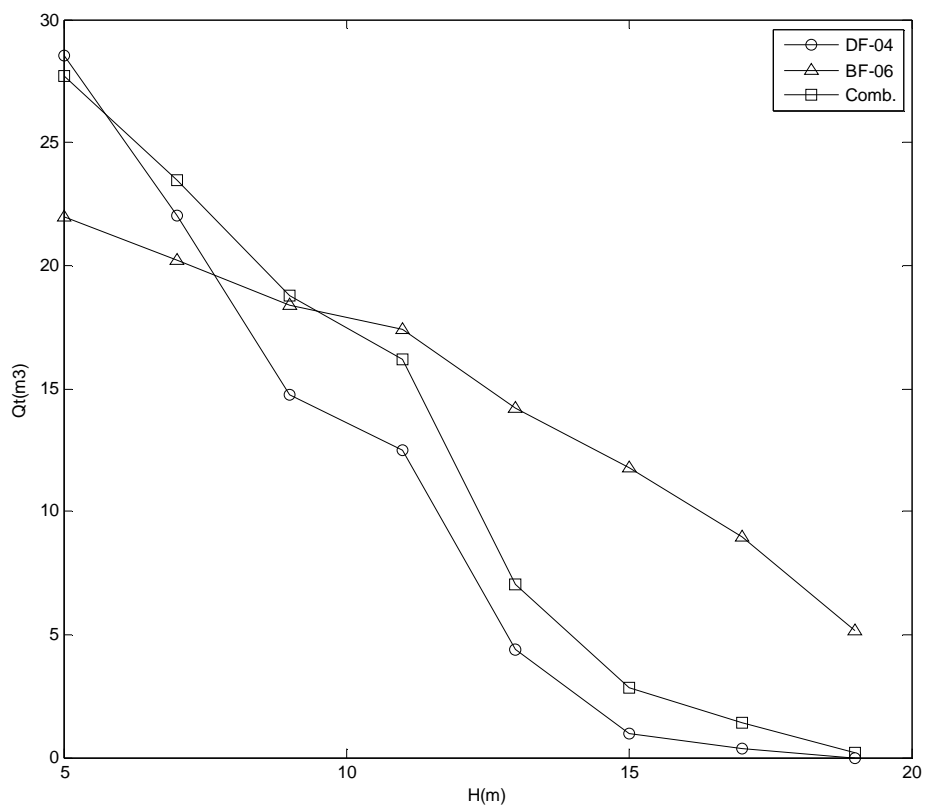

Fig. 8 Water output $\left(\mathrm{m}^{3} /\right.$ day) under $\mathrm{S}_{\max }=700 \mathrm{~W} / \mathrm{m}^{2}$

As can be found in Fig. 8:

1) The water output per day of three kinds of pump systems were decreased significantly compared to the water output per day under the insolation condition $S_{\max }=1000 \mathrm{~W} / \mathrm{m}^{2}$. 
2) When pump head is lower than $8 \mathrm{~m}$, the water output per day of DF-04 system is higher than BF-06 system. And the proposed system has more water output per day than BF-06 system when pump head is lower than $10 \mathrm{~m}$. The water output per day of the proposed system is better than DF-04 system in the whole range of pump head.

\section{Conclusions}

This paper established mathematical models of PV pumping system, carried out the simulation study and analysis to the system efficiency and water output per day of three kinds of water pump system under different maximum insolation, pump switch insolation and pump head, and finally deduced some conclusions which could be referred by system designers:

1) When the maximum insolation and distribution is confirmed, the pump head influence the system efficiency and water output per day remarkably. When the pump head is determined, the water pump system which corresponds to the maximum system efficiency or the maximum water output per day should be preferred.

2) The water output per day of the proposed system is higher than DF-04 system in the whole range of pump heads. The BF-06 system or the proposed system should be chosen scientifically according to pump head.

3) Different maximum insolation infects the pump head corresponding to maximum water output per day significantly. So maximum insolation distribution during a period of time, such as month, year etc. should be well considered.

4) When the maximum insolation is confirmed, under different pump head, the pump switch isolation has certain influence to the water output per day of the proposed system. The pump switch insolation should be set cautiously refer to optimize pump head to maximum water output per day.

\section{References}

[1] A Woodhoo, M Iruarrizaga-Lejarreta, N Beraza, JL García-Rodríguez, N Embade. The feasibility study of solar irrigation: Economical comparison between diesel and photovoltaic water pumping systems for different crops[C]. International Conference on Electrical Information and communication Technology,2014,7(1):1-5.

[2] BD Vick, RN Clark. Experimental investigation of solar powered diaphragm and helical pumps [J]. Solar Energy 2011,85(5):945-954.

[3] Lei Xie, Shijie Yu, Fei Wang, Liming Ma. The experimental and simulation research on system efficiency of photovoltaic pumping system[C]. The 4th IEEE Conference on Industrial Electronics and Applications. 25-27 May 2009: 2335-2339.

[4] Borowy BS and Salameh ZM. Methodology for optimally sizing the combination of a battery bank and PV array in a wind/PV hybrid system. IEEE Transactions on Energy Conversion, 1996, 11(2):367-373.

[5] A. A. Ghoneim. Design optimization of photovoltaic powered water pumping systems[J]. Energy Conversion and Management. 2006,6:1449-1463.

[6] Jiangmen Ruirong pump industry Co. Ltd. "Datasheet of 4' submersible pumps of R95 series" (http://www.ruirong.com/cn/4-SUBMERSIBLE-PUMP.htm), 2016. 